\title{
DETERMINAÇÃO DO FLUXO DE SEIVA NA CANA-DE-AÇÚCAR PELO MÉTODO DO BALANÇO DE ENERGIA CAULINAR ${ }^{1}$
}

\section{DAVI BOEHRINGER ${ }^{2}$, SÉRGIO ZOLNIER ${ }^{3}$, ARISTIDES RIBEIRO ${ }^{4}$, ANTONIO J. STEIDLE NETO ${ }^{5}$}

\begin{abstract}
RESUMO: Medições do fluxo de seiva são importantes não somente no campo da agrometeorologia, mas também em estudos de natureza fisiológica para identificação de doenças vasculares e pesquisas destinadas à quantificação do movimento de água no sistema solo-planta-atmosfera. Este trabalho foi realizado com o objetivo de avaliar o desempenho do método do balanço de energia, a partir da inserção de um minirresistor no centro do colmo de plantas de cana-de-açúcar, visando à determinação do fluxo de seiva sob condições ambientais distintas, utilizando-se de medições gravimétricas como padrão comparativo. $\mathrm{O}$ experimento foi conduzido em uma casa de vegetação não climatizada, localizada na área experimental da Universidade Federal de Viçosa, Viçosa - MG. Com base no erro médio de estimativa (MBE), verificou-se que o método do balanço de energia apresentou desempenho satisfatório para a determinação da transpiração da cana-de-açúcar, tanto em condições de céu nublado como parcialmente nublado, tendo subestimado os valores gravimétricos em apenas 3,0 e 6,5 $\mathrm{g}_{\text {planta }}{ }^{-1} \mathrm{~h}^{-1}$, respectivamente. Em termos percentuais, esses valores correspondem a 4 e 5\% da transpiração máxima da cana-de-açúcar.
\end{abstract}

PALAVRAS-CHAVE: transpiração, Saccharum officinarum, eficiência do uso de água, disponibilidade hídrica, irrigação.

\section{SUGARCANE SAP FLOW DETERMINATION BY THE ENERGY BALANCE METHOD}

ABSTRACT: Sap flow measurements are important not only in the agrometeorology field, but also in studies of physiological nature to identify vascular diseases and researches concerned with quantification of water movement in soil-plant-atmosphere system. This study was carried out to evaluate the performance of the energy balance method, implemented by insertion of a miniresistor in the stem center of sugarcane plants, in order to determine the sap flow under distinct environment conditions, utilizing gravimetric measurements as comparative standard. The experiment was conducted in a non-environment controlled greenhouse, located at the Federal University of Viçosa, Viçosa-MG. Based on the mean bias error (MBE), it was verified that the energy balance method presented satisfactory results to determine sugarcane transpiration, both in cloudy and partly cloudy sky conditions, underestimating gravimetric values by only 3.0 and $6.5 \mathrm{~g} \mathrm{plant}^{-1} \mathrm{~h}^{-1}$, respectively. In percentage terms, these values correspond to 4 and $5 \%$ of the maximum sugarcane transpiration.

KEYWORDS: transpiration, Saccharum officinarum, water use efficiency, water requirement, irrigation.

\footnotetext{
${ }^{1}$ Extraído da Dissertação de Mestrado do primeiro autor. Bolsa de estudos concedida pelo CNPq e auxílio financeiro para a execução do projeto de pesquisa proveniente da FAPEMIG.

${ }^{2}$ Eng $^{\mathrm{O}}$ Agrônomo, Doutorando, Departamento de Engenharia Agrícola, DEA/UFV, Viçosa - MG.

${ }^{3}$ Eng $^{\mathrm{o}}$ Agrícola, Prof. Doutor, Departamento de Engenharia Agrícola, DEA/UFV, Viçosa - MG.

${ }^{4}$ Eng $^{\mathrm{O}}$ Agrônomo, Prof. Doutor, Departamento de Engenharia Agrícola, DEA/UFV, Viçosa - MG.

${ }^{5}$ Eng $^{\mathrm{o}}$ Agrícola, Prof. Doutor, Câmpus Sete Lagoas, CSL/UFSJ, Sete Lagoas - MG.

Recebido pelo Conselho Editorial em: 8-12-2011
}

Aprovado pelo Conselho Editorial em: 24-9-2012 


\section{INTRODUÇÃO}

A cana-de-açúcar desempenha papel de extrema importância para a economia brasileira, com produção de álcool e de açúcar para os mercados interno e externo. Em 2010, a produção brasileira foi de 717,5 milhões de toneladas, correspondente a uma produtividade média de 79,04 t ha-1 (IBGE, 2011).

Limitações para expansão de áreas produtoras de cana-de-açúcar têm levado à necessidade de pesquisas que contemplem o aumento de produtividade e, principalmente, a otimização do uso da água, seja pela aplicação de volumes mais adequados (IGBADUN et al., 2006), seja pela utilização de sistemas de irrigação mais eficientes (RAJAK et al., 2006) e aprimoramento do manejo de irrigação (JALOTA et al., 2006). Sob condições de cultivo em sequeiro, estudos sobre o consumo de água da cana-de-açúcar tornam-se importantes em razão de existir uma variação genotípica de resposta ao estresse hídrico por diferentes variedades (SMIT \& SINGELS, 2006; TEJERA et al., 2007). Em decorrência disso, há grande interesse pelo uso de métodos que permitam a determinação isolada da transpiração e não da perda total de água pela cultura, que inclui a evaporação na superfície do solo ou substrato (ZOLNIER et al., 2003). Neste sentido, nos últimos anos, houve aumento expressivo no número de pesquisas científicas relacionadas à medição da transpiração por meio de técnicas térmicas, a partir da determinação do movimento de seiva no interior do caule.

Existem três principais métodos térmicos para medição do fluxo de seiva: dissipação de calor, balanço de energia e pulso de calor (REIS et al., 2009). No Brasil, a aplicabilidade desses métodos para a determinação da transpiração foi avaliada em laranjeira e mangueira (VELLAME et al., 2009; VELLAME et al., 2010); cafeeiro (PIMENTEL et al., 2010); citros (GIRARDI et al., 2010); lima-ácida (DELGADO-ROJAS et al., 2007); seringueira (DELGADO-ROJAS et al., 2006) e mudas de limoeiro (TREJO-CHANDIA et al., 1997). Adicionalmente, estes métodos foram utilizados em estudos dos efeitos ambientais sobre a transpiração (REIS et al., 2009; VASCONCELOS, 2008; COSTA et al., 2007), na detecção de estresse hídrico (GOMIDE et al., 2005) e na avaliação de obstruções do xilema da laranjeira, causadas pela clorose variegada dos citros (MACHADO et al., 2006). No entanto, poucos trabalhos relacionados à aplicabilidade de técnicas térmicas em plantas de cana-de-açúcar (Saccharum officinarum) foram publicados no Brasil (ALVES, 1999) e no exterior (CHABOT et al., 2005).

A base teórica do método do balanço de energia foi apresentada e discutida em revisões científicas (MARIN et al., 2008; ALLEN et al., 2011). A técnica consiste na aplicação contínua de calor por meio da circulação de corrente elétrica através de resistências dispostas na superfície ou no interior do caule de uma planta. A diferença de temperatura acima e abaixo da fonte de aquecimento é usada para a medição do fluxo de seiva (MARIN et al., 2008).

O balanço de energia tem sido aplicado com bons resultados para estimativa do fluxo de seiva de plantas herbáceas com diâmetro de caule inferior a $2 \mathrm{~cm}$ (SAKURATANI, 1981). No entanto, à medida que o diâmetro aumenta, dificuldades associadas ao isolamento térmico do sistema de medição e contato da fonte de aquecimento com o caule promovem erros na determinação dos componentes radial e axial do balanço de energia, principalmente durante períodos de baixa transpiração (HERZOG et al., 1997; TREJO-CHANDIA et al., 1997; MARIN et al., 2008). Assim, para plantas com diâmetros maiores, esta técnica tem sido substituída pelo método da dissipação de calor ou pulso de calor, com a introdução da fonte de aquecimento no caule, apesar de ser uma solução invasiva para o processo de medição (COELHO et al., 2012; DELGADO-ROJAS et al., 2007).

Estudos que utilizam métodos de medição do fluxo de seiva são de grande relevância para identificar variedades de cana-de-açúcar resistentes ao estresse hídrico. Além disso, a medição do fluxo de seiva é importante não somente no campo da agrometeorologia, mas também em estudos de natureza fisiológica para identificação de doenças vasculares e estudos destinados à quantificação do movimento de água no sistema solo-planta-atmosfera. 
Este trabalho foi realizado com o objetivo de avaliar o desempenho do método do balanço de energia para a determinação do fluxo de seiva da cana-de-açúcar sob condições ambientais distintas, a partir da aplicação de calor com um minirresistor introduzido no centro do segmento de caule da planta. O desempenho deste método foi avaliado com base em medições gravimétricas de transpiração que foram utilizadas como padrão comparativo.

\section{MATERIAL E MÉTODOS}

\section{Local e técnicas de cultivo da cana-de-açúcar}

O trabalho foi conduzido em uma casa de vegetação não climatizada, localizada na área experimental do Setor de Meteorologia Agrícola, pertencente ao Departamento de Engenharia Agrícola da Universidade Federal de Viçosa, Viçosa - MG (latitude $20^{\circ} 45^{\prime}$ S; longitude $42^{\circ} 52^{\prime}$ O; altitude de $690 \mathrm{~m}$ ).

Para a utilização total do espaço interior da casa de vegetação, foram cultivadas 192 plantas de cana-de-açúcar (cv. RB867515) em vasos plásticos com capacidade unitária para 15 L, os quais foram dispostos em 8 fileiras. Os vasos foram preenchidos com $10 \mathrm{~L}$ de substrato comercial (Bioplant, Nova Ponte - MG), composto por casca de pinus, agentes agregantes, vermiculita, fibra de coco e complementos minerais (NPK e micronutrientes). No fundo de cada vaso de cultivo, foi perfurado um orifício circular para permitir o escoamento do excesso da água de irrigação ou da solução de fertirrigação, após sua drenagem pelo substrato.

A condutividade elétrica (CE) da solução drenada nos vasos foi monitorada por meio de um condutivímetro (modelo CDH-42, Omega, Stamford, CT, USA), sendo mantida entre 2,0 e $3,0 \mathrm{dS} \mathrm{m}^{-1}$ por meio de fertirrigações periódicas. A solução nutritiva utilizada nas fertirrigações foi preparada manualmente em um reservatório plástico de $100 \mathrm{~L}$, utilizando-se de fertilizante solúvel em água (Peters Professional 20-20-20 GP, Marysville, OH, USA). As plantas foram irrigadas e fertirrigadas por gotejamento, com duração e frequência dos eventos controladas por temporizador.

Após o início do perfilhamento, as brotações laterais foram removidas periodicamente, mantendo-se apenas um colmo por planta, com tutoramento exclusivo para sustentar o crescimento vertical e evitar o tombamento causado pelo próprio peso. Para minimizar a evaporação, a superfície do substrato de cultivo foi revestida com filme plástico de polietileno. Dessa forma, alterações na massa do conjunto vaso-substrato-planta foram atribuídas exclusivamente à transpiração.

\section{Sistema de aquisição de dados}

Os sinais analógicos dos sensores responsáveis pelas medições das variáveis meteorológicas, no interior da casa de vegetação, foram transmitidos a uma placa de aquisição de dados (modelo CYDAS 1602HR, CyberResearch, Branford, CT, USA), instalada em um microcomputador e gerenciada por um programa computacional especialmente desenvolvido para esta finalidade. $\mathrm{O}$ programa foi implementado usando a plataforma de programação $C++$ Builder (versão 6.0) no ambiente Windows. A temperatura e a umidade relativa do ar foram medidas por meio de um sensor conjugado (modelo Humitter 50Y, Vaisala Inc., Woburn, MA, USA), que foi instalado no interior de um tubo de PVC branco, onde o ar foi aspirado por meio de uma ventoinha. A estrutura em PVC foi projetada para impedir a incidência direta da radiação solar sobre os sensores e, concomitantemente, garantir uma taxa de ventilação adequada.

A radiação solar global foi medida por meio de um piranômetro (modelo LI-200SA, LI-COR, Lincoln, NE, USA), que foi fixado na parte superior da estrutura de PVC. Inicialmente, os sensores foram dispostos a 1,5 m da superfície do solo, e, posteriormente, o nível de posicionamento destes foi alterado periodicamente, de acordo com o crescimento das plantas, com o propósito de mantê-los acima do dossel vegetativo da cultura. 
As temperaturas da seiva, da superfície do colmo e do centro da manta de isolamento térmico foram determinadas por meio de termopares do tipo cobre-constantan (Tipo T, Omega, Stamford, CT, USA), conectados a módulos seriais de entradas analógicas (modelo LR-7018, LR Informática Industrial, Porto Alegre - RS). Por outro lado, o controle da fonte de aquecimento, constituída de um minirresistor de $18 \Omega$, foi efetuado por meio do módulo de saídas digitais a relés (modelo LR7067D, LR Informática Industrial, Porto Alegre - RS).

As tensões elétricas aplicadas aos terminais resistivos dos dispositivos de aquecimento foram obtidas, separadamente para cada planta, com o módulo serial de entradas analógicas (modelo LR-7017F, LR Informática Industrial, Porto Alegre - RS), o qual foi configurado para atuar na faixa de $\pm 10 \mathrm{~V}$. Em decorrência do regulador de tensão elétrica da fonte de alimentação, o valor aplicado esteve constante ao longo do período experimental $(2,970 \pm 0,001 \mathrm{~V})$. O valor da tensão elétrica medida foi multiplicado pelo valor do resistor de precisão para a determinação da potência elétrica aplicada pelo sistema de medição do fluxo de seiva da cana-de-açúcar. Todos os dados medidos, em intervalos de tempo de $1 \mathrm{~min}$, foram transmitidos para o microcomputador por meio do protocolo serial (RS-232).

A transpiração da cana-de-açúcar foi monitorada em intervalos de tempo de 1 min, no período de 21 a 30 de outubro de 2009, entre 7 e 19 h, por meio de balanças de precisão (Tecnal, Piracicaba$\mathrm{SP}$ ), com capacidade individual de $12 \mathrm{~kg}$ e precisão de $0,1 \mathrm{~g}$ (método gravimétrico).

\section{Método do balanço de energia}

A técnica do balanço de energia consiste na aplicação contínua de uma tensão elétrica nos terminais de um resistor, de forma que a potência $(\mathrm{P})$ seja dissipada na forma de calor. A fonte de aquecimento foi instalada no interior de um segmento vegetal (colmo), sendo que o balanço de energia foi obtido pela diferença entre o somatório das entradas e saídas de calor (eq.(1)):

$$
\frac{\mathrm{d}}{\mathrm{d} \theta}\left(\mathrm{m}_{\mathrm{TV}} \mathrm{c}_{\mathrm{pTV}} \mathrm{t}_{\mathrm{TV}}\right)=\mathrm{P}-\left(\mathrm{Q}_{\mathrm{C}}+\mathrm{Q}_{\mathrm{S}}\right)
$$

em que,

$\mathrm{d} / \mathrm{d} \theta$ - elemento infinitesimal de tempo;

$\mathrm{m}_{\mathrm{TV}}$ - massa do tecido vegetal do segmento de caule, $\mathrm{kg}$;

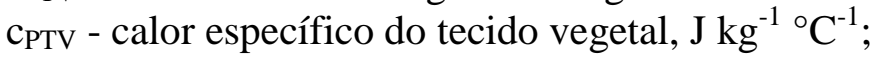

$\mathrm{t}_{\mathrm{TV}}$ - temperatura média do tecido vegetal, ${ }^{\circ} \mathrm{C}$;

$\mathrm{P}$ - potência elétrica aplicada ao segmento de caule, $\mathrm{W}$;

$\mathrm{Q}_{\mathrm{C}}$ - calor transferido por condução através do material vegetal e, posteriormente, através da manta de isolamento térmico, considerando-se, concomitantemente, as direções axial e radial, $\mathrm{W}$, e

$\mathrm{Q}_{\mathrm{S}}$ - calor transferido pelo transporte de seiva na direção axial, W.

Em condições de regime permanente e para plantas que possuem apenas um pequeno volume de seiva armazenada no tecido vegetal, a taxa de variação da temperatura no tecido vegetal $\left(\mathrm{dt}_{\mathrm{TV}} / \mathrm{d} \theta\right)$ torna-se desprezível, e, assim, o lado esquerdo da eq.(1) pode ser anulado. No entanto, para a cana-de-açúcar, esse termo precisa ser determinado, e esta equação deve ser reescrita como:

$$
\mathrm{Q}_{\mathrm{S}}=\mathrm{P}-\left(\mathrm{Q}_{\mathrm{C}}+\mathrm{Q}_{\mathrm{ARM}}\right)
$$

em que, $\mathrm{Q}_{\mathrm{ARM}}$ é a quantidade de calor armazenado no segmento do caule, W.

Portanto, o fluxo de seiva $\left(\mathrm{F}_{\mathrm{S}}\right)$, expresso $\mathrm{em}_{\mathrm{g} \mathrm{h}} \mathrm{h}^{-1}$, foi determinado pela eq.(3):

$$
\mathrm{F}_{\mathrm{S}}=\frac{3600 \mathrm{Q}_{\mathrm{S}}}{\mathrm{C}_{\mathrm{pTV}}\left(\mathrm{t}_{\mathrm{S}}-\mathrm{t}_{\mathrm{I}}\right)}=\frac{3600\left[\mathrm{P}-\left(\mathrm{Q}_{\mathrm{C}}+\mathrm{Q}_{\mathrm{ARM}}\right)\right.}{\mathrm{C}_{\mathrm{pTV}}\left(\mathrm{t}_{\mathrm{S}}-\mathrm{t}_{\mathrm{I}}\right)}=\frac{3600\left[\mathrm{U}^{2} / \mathrm{R}-\left(\mathrm{Q}_{\mathrm{C}}+\mathrm{Q}_{\mathrm{ARM}}\right)\right]}{\mathrm{C}_{\mathrm{pTV}}\left(\mathrm{t}_{\mathrm{S}}-\mathrm{t}_{\mathrm{I}}\right)}
$$


em que,

$t_{S}$ e $t_{I}$-temperaturas médias das seções superiores e inferiores do segmento de caule, respectivamente, ${ }^{\circ} \mathrm{C}$;

$\mathrm{U}$ - tensão elétrica aplicada aos terminais resistivos, $\mathrm{V}$, e

$\mathrm{R}$ - resistência elétrica do dispositivo de aquecimento, $\Omega$.

O calor transferido nas direções axial e radial foi estimado a partir da equação de Fourier. Neste experimento, os dois fluxos foram combinados, a partir do coeficiente global de transferência de calor por condução, que foi determinado, diariamente, no horário imediatamente anterior ao nascer do sol, quando o fluxo de seiva se torna praticamente nulo.

Nas plantas utilizadas nas medições, foram inseridos dois termopares no centro do colmo, sendo posicionados equidistantes $1,5 \mathrm{~cm}$ abaixo e acima da fonte de aquecimento. Além disso, foram instalados dois termopares na superfície do colmo, posicionados $1,5 \mathrm{~cm}$ abaixo e acima do nível de aquecimento. Finalmente, com o objetivo de medir o fluxo radial de calor por condução, foi instalado um termopar no centro da manta de isolamento térmico, posicionado ao nível da fonte de aquecimento. A ilustração experimental deste procedimento e a representação esquemática dos componentes do balanço de energia, assim como a disposição dos termopares, estão apresentadas nas Figuras 1 e 2, respectivamente.

Foram conduzidas medições em dias com condições de céu parcialmente nublado e nublado, utilizando três plantas de cana-de-açúcar, em delineamento completamente casualizado. Os valores do fluxo de seiva foram comparados com os de transpiração obtidos, em base horária e diária, por meio do método gravimétrico, considerado padrão para teste do método do balanço de energia.

Para a avaliação do método do balanço de energia, foram calculados índices estatísticos de precisão (coeficiente de correlação) e de exatidão (índice de concordância "d"). Adicionalmente, foram calculados os seguintes erros estatísticos, conforme sugerido por JACOVIDES \& KONTOYIANNIS (1995): erro médio de estimativa (MBE) e raiz quadrada do erro médio de estimativa (RMSE).
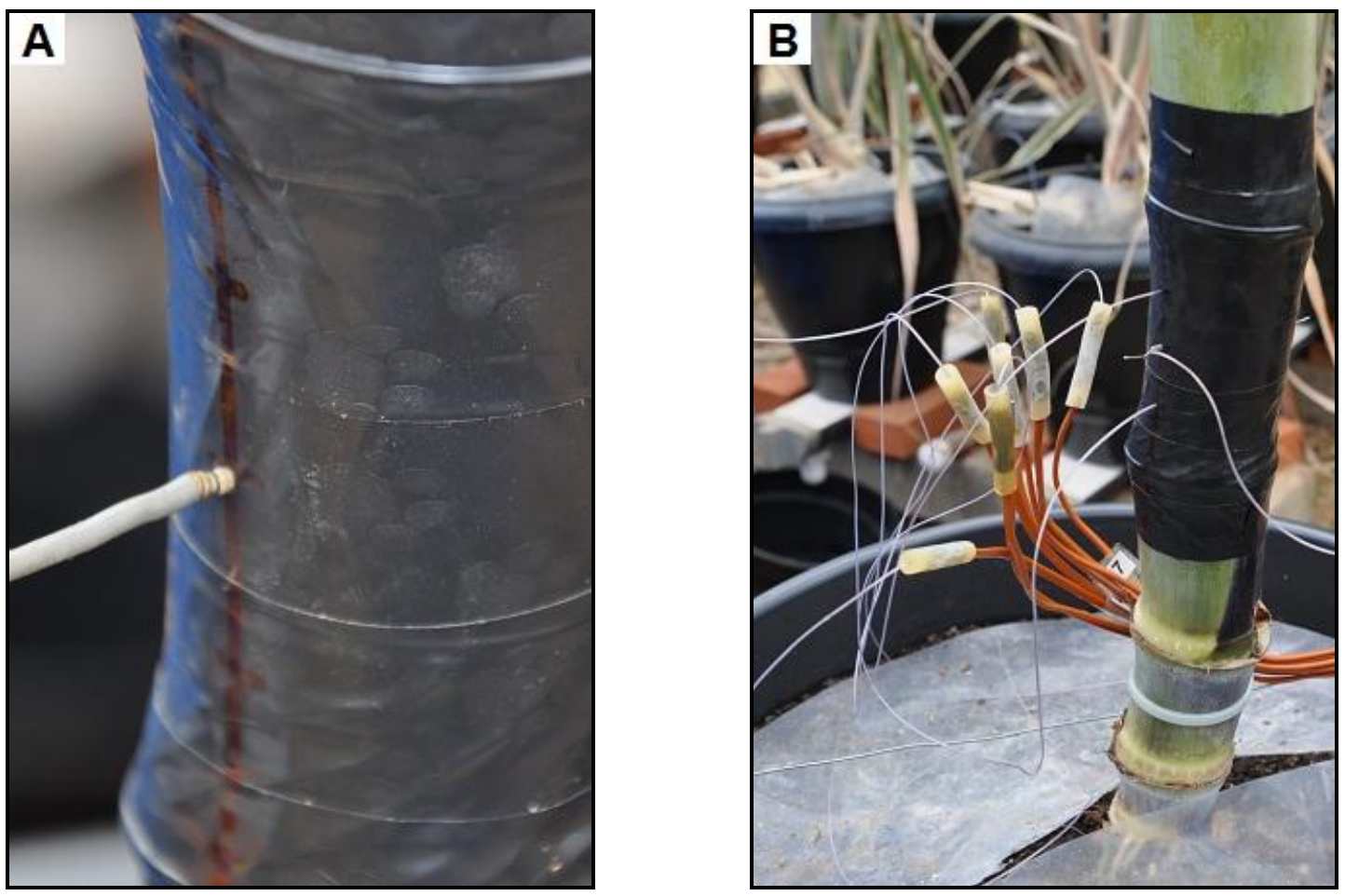

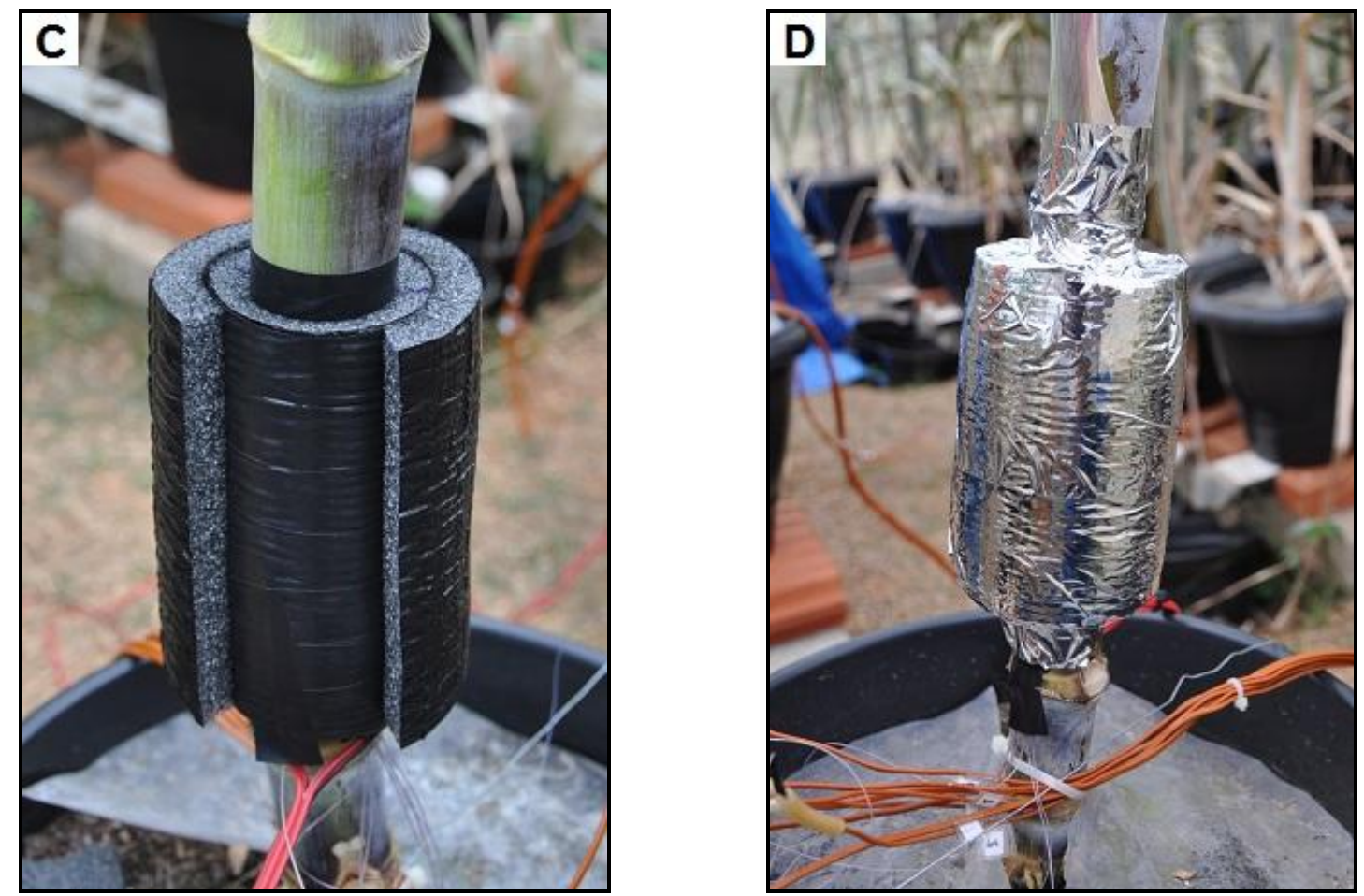

FIGURA 1. Inserção do minirresistor no colmo de uma planta de cana-de-açúcar (A), e dos termopares acima e abaixo da fonte de aquecimento (B); isolamento térmico (C), e revestimento do sistema de medição com fita de alumínio (D). Insertion of the miniresistor in a sugarcane stem (A) and thermocouples above and below the heating source $(B)$; thermal insulation $(C)$ and covering of the measurement system with aluminum tape (D).
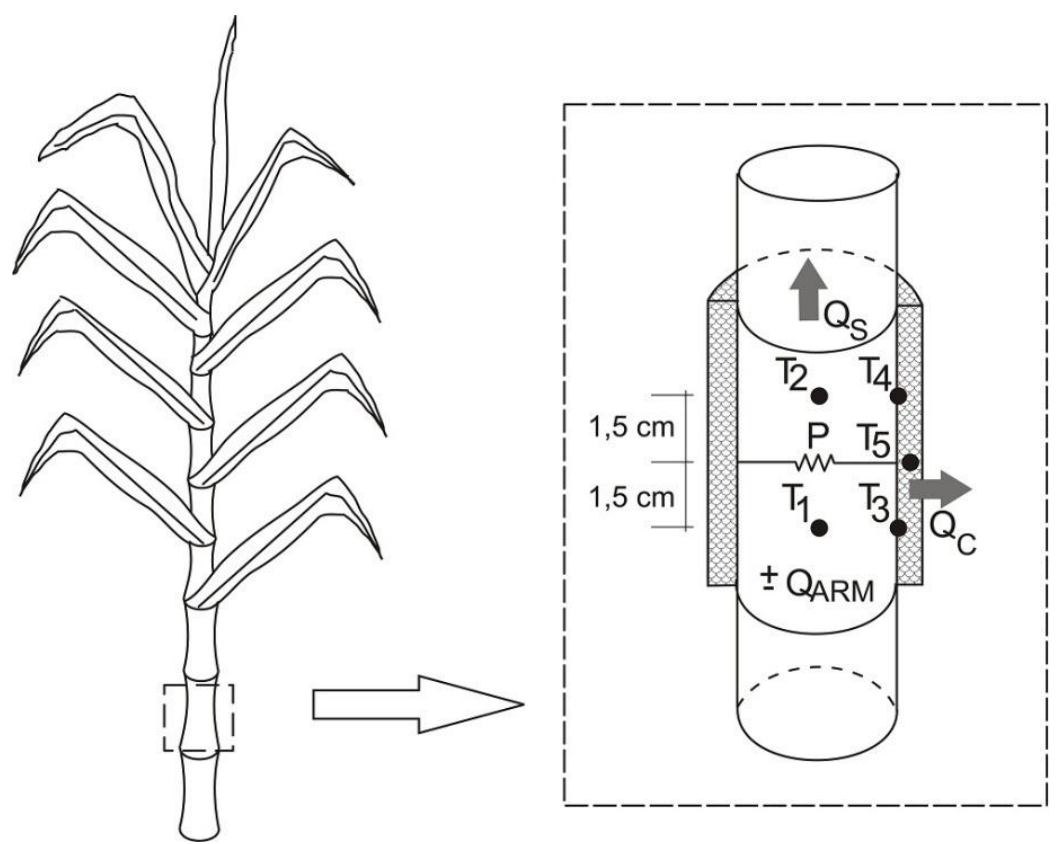

FIGURA 2. Representação esquemática dos componentes do balanço de energia ( $\mathrm{P}, \mathrm{Q}_{\mathrm{C}}, \mathrm{Q}_{\mathrm{S}}$ e $\left.\mathrm{Q}_{\mathrm{ARM}}\right)$, conforme descrito na Equação 2. Os termopares foram dispostos no centro (1 e 2) e na superfície ( 3 e 4) do colmo da cana-de-açúcar e, adicionalmente, no centro do isolamento térmico (5) ao nível do minirresistor. Schematic representation of the energy balance componentes ( $P, Q_{C}, Q_{S}$ e $\left.Q_{A R M}\right)$, as described in Equation 2. The thermocouples were placed in the center ( 1 and 2 ) and in the sugarcane stem surface ( 3 and 4$)$, and additionally, in the center of the thermal insulation (5) at the miniresistor position. 


\section{RESULTADOS E DISCUSSÃO}

\section{Condições de céu parcialmente nublado}

$\mathrm{Na}$ Figura 3A, são apresentadas as variações da radiação solar global $\left(\mathrm{R}_{\mathrm{g}}\right)$, da temperatura do ar $\left(\mathrm{T}_{\mathrm{ar}}\right)$ e do déficit de pressão de saturação de vapor d'água no ar $\left(\mathrm{DPV}_{\mathrm{ar}}\right)$, para 21 de outubro de 2009, sob condições de céu parcialmente nublado, em Viçosa-MG. As alterações horárias da transpiração da cana-de-açúcar, obtidas pelos métodos gravimétrico e balanço de energia, correspondentes às condições meteorológicas deste dia, estão apresentadas na Figura 3B. Constatou-se que o balanço de energia apresentou resultados satisfatórios para a quantificação da transpiração da cana-de-açúcar, acompanhando de maneira mais evidente a variação da radiação solar global ao longo do período diurno. Tal fato também foi verificado por OLIVEIRA et al. (2009), VASCONCELOS (2008) e ZHANG et al. (2011).
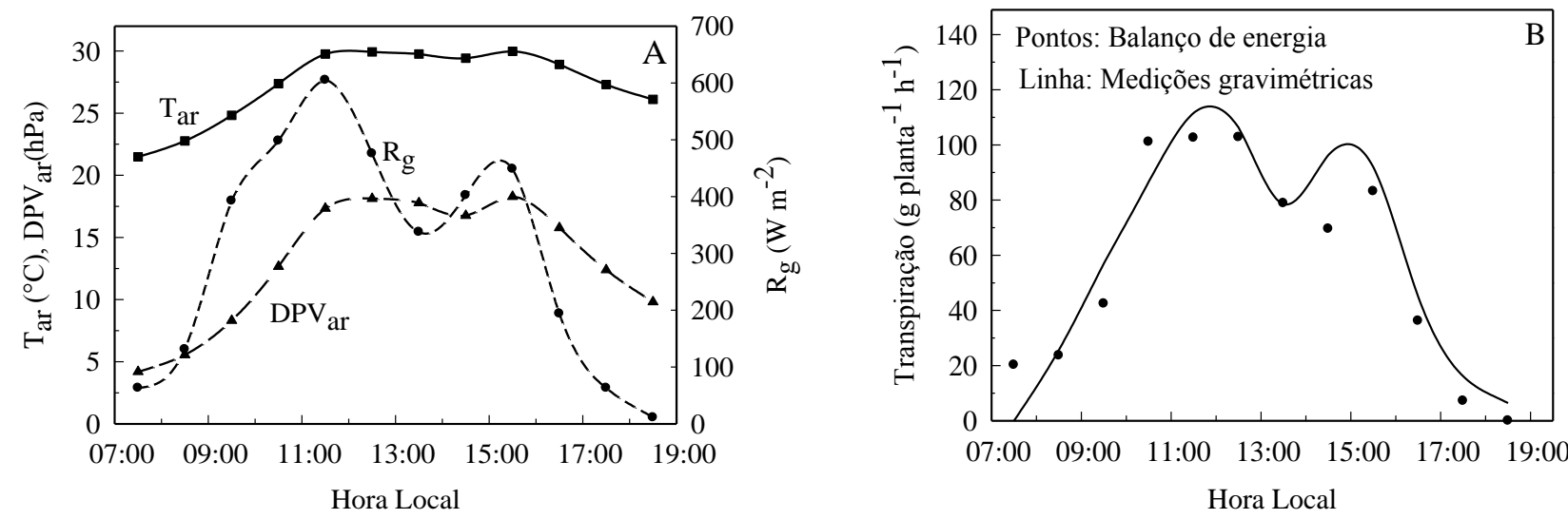

FIGURA 3. Variações típicas da radiação solar global $\left(\mathrm{R}_{\mathrm{g}}\right)$, temperatura do ar $\left(\mathrm{T}_{\mathrm{ar}}\right)$ e déficit de pressão de saturação de vapor d'água no ar $\left(\mathrm{DPV}_{\mathrm{ar}}\right)$, para 21 de outubro de 2009, sob condições de céu parcialmente nublado, em Viçosa - MG (A), e alterações correspondentes da transpiração horária da cana-de-açúcar (B). Typical variations of global solar radiation $\left(R_{g}\right)$, air temperature $\left(T_{a r}\right)$ and air saturation vapor pressure deficit $\left(\mathrm{DPV}_{\mathrm{ar}}\right)$, on October 21,2009 , under partly cloudy sky conditions in Viçosa-MG (A) and corresponding alterations of the hourly sugarcane transpiration (B).

Neste dia, a transpiração máxima da cana-de-açúcar foi superior a $100 \mathrm{~g}$ planta ${ }^{-1} \mathrm{~h}^{-1}$, quando os valores aproximados de $\mathrm{T}_{\mathrm{ar}}, \mathrm{DPV}_{\mathrm{ar}}$ e $\mathrm{R}_{\mathrm{g}}$ foram, respectivamente, $30{ }^{\circ} \mathrm{C}, 18 \mathrm{hPa}$ e $600 \mathrm{~W} \mathrm{~m} \mathrm{~m}^{-2}$. Especificamente entre 7 e 8 e entre 10 e 11 horas, o método do balanço de energia superestimou a transpiração medida pelo método gravimétrico, enquanto na maior parte do período diurno a transpiração foi ligeiramente subestimada.

\section{Condições de céu nublado}

As variações típicas da radiação solar global $\left(\mathrm{R}_{\mathrm{g}}\right)$, da temperatura do ar $\left(\mathrm{T}_{\mathrm{ar}}\right)$ e do déficit de pressão de saturação de vapor d'água no ar $\left(\mathrm{DPV}_{\mathrm{ar}}\right)$, para 22 de outubro de 2009, sob condições de céu nublado, em Viçosa - MG, são apresentadas na Figura 4A. Paralelamente, pode-se observar a influência das condições meteorológicas sobre a transpiração neste mesmo dia (Figura 4B).

Verificou-se que, durante o período diurno, a transpiração determinada pelo método gravimétrico variou de 5 a $32 \mathrm{~g}_{\text {planta }}{ }^{-1} \mathrm{~h}^{-1}$, entre o início do período da manhã e 11 horas, aproximadamente. A partir desse horário, a transpiração sofreu oscilações de acordo com a variação correspondente na radiação solar global. No entanto, com exceção do início da manhã, a transpiração determinada pelo método do balanço de energia foi subestimada a partir das 11 horas, mas as diferenças foram da ordem de, apenas, $5 \mathrm{~g} \mathrm{planta}^{-1} \mathrm{~h}^{-1}$. 

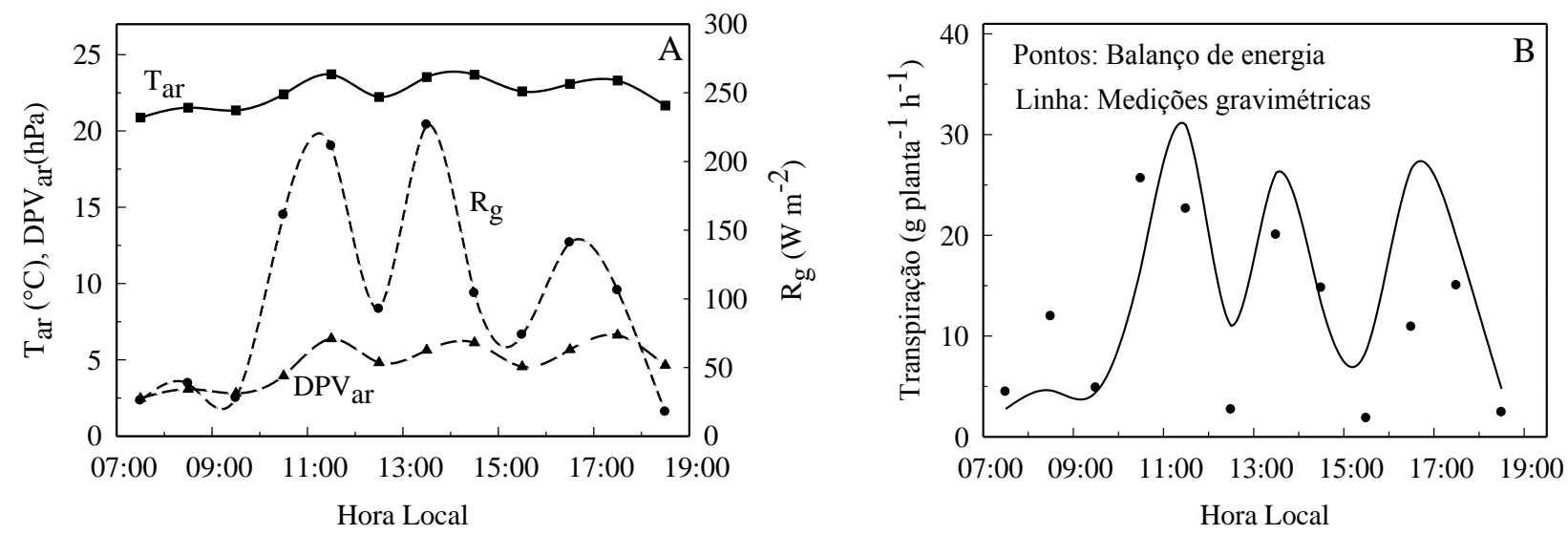

FIGURA 4. Variações típicas da radiação solar global $\left(\mathrm{R}_{\mathrm{g}}\right)$, temperatura do ar $\left(\mathrm{T}_{\mathrm{ar}}\right)$ e déficit de pressão de saturação de vapor d'água no ar $\left(\mathrm{DPV}_{\mathrm{ar}}\right)$, para 22 de outubro de 2009, sob condições de céu nublado, em Viçosa-MG (A), e alterações correspondentes da transpiração horária da cana-de-açúcar (B). Typical variations of global solar radiation $\left(R_{\mathrm{g}}\right)$, air temperature $\left(T_{\mathrm{ar}}\right)$ and air saturation vapor pressure deficit $\left(\mathrm{DPV}_{\mathrm{ar}}\right)$, on October 22, 2009 under cloudy sky conditions in Viçosa-MG (A) and corresponding alterations of the hourly sugarcane transpiration (B).

\section{Relação entre transpiração e fluxo de seiva}

Na Figura 5A, são apresentados os valores horários do fluxo de seiva obtidos pelo método do balanço de energia durante quatro dias, sob condições de céu nublado. De maneira análoga, na Figura 5B, são mostradas medições realizadas também durante quatro dias, porém em condições de céu parcialmente nublado. Os dados referentes às duas condições de nebulosidade, ou seja, nublado e parcialmente nublado, foram também avaliados conjuntamente, em escala horária, sendo apresentados na Figura 6A. Adicionalmente, os valores diários do fluxo de seiva para todos os dias de medição são apresentados na Figura 6B.
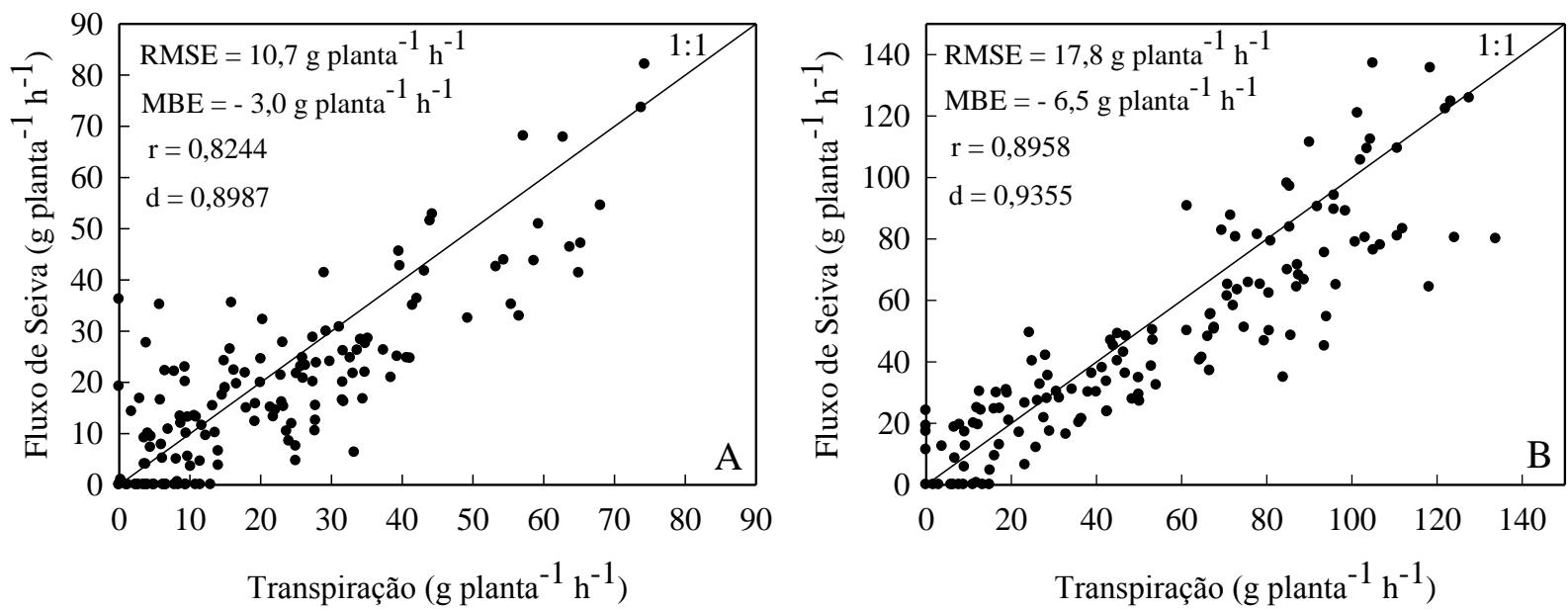

FIGURA 5. Relação entre transpiração (método gravimétrico) e fluxo de seiva (método do balanço de energia) da cana-de-açúcar, referente a medições realizadas em quatro dias nublados (A) e em quatro dias parcialmente nublados (B). Relation between sugarcane transpiration (gravimetric method) and sap flow (energy balance method), whose measurements were conducted in four cloudy (A) and four partly cloudy days (B).

Com base no indicador estatístico MBE, verificou-se que o método do balanço de energia apresentou desempenho satisfatório para a determinação da transpiração da cana-de-açúcar, tanto em condições de céu nublado como de parcialmente nublado, tendo subestimado a transpiração em 
apenas 3,0 e 6,5 g planta ${ }^{-1} \mathrm{~h}^{-1}$, respectivamente. Em termos percentuais, esses valores correspondem a 4 e 5\% da transpiração máxima da cana-de-açúcar, em condições de céu nublado e parcialmente nublado, respectivamente. Quando as duas condições de nebulosidade são combinadas, a transpiração foi subestimada em $4,7 \mathrm{~g}_{\text {planta }}{ }^{-1} \mathrm{~h}^{-1}$, representando aproximadamente $3 \%$ da transpiração máxima das plantas (Figura 6A).
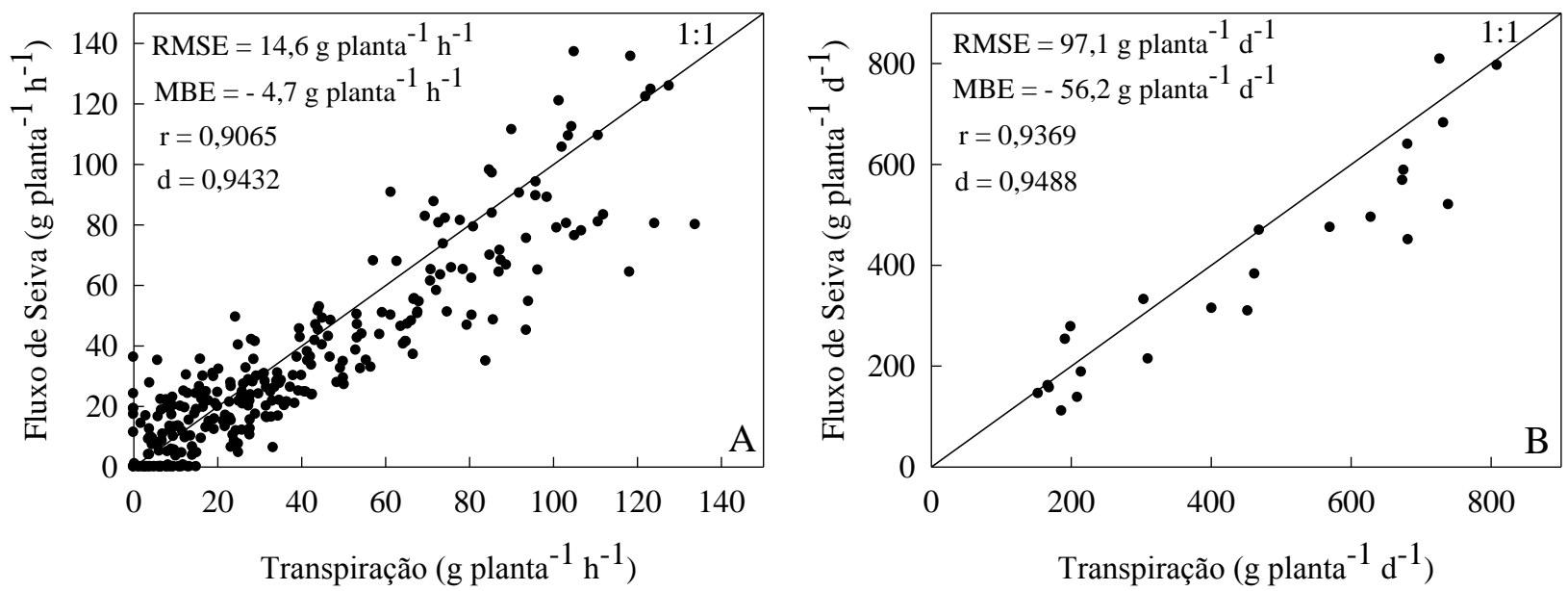

FIGURA 6. Relação entre transpiração (método gravimétrico) e fluxo de seiva (método do balanço de energia) da cana-de-açúcar, referente a medições realizadas em dias nublados e parcialmente nublados, em escala horária (A) e em escala diária (B). Relation between sugarcane transpiration (gravimetric method) and sap flow (energy balance method), whose hourly (A) and daily (B) measurements were carried out on cloudy and partly cloudy days.

Em trabalhos realizados com plantas de tomate, milho e mudas de limoeiro, comparações entre valores do fluxo de seiva, determinado pelo método do balanço de energia, e da transpiração obtida com balanças de precisão têm mostrado diferenças inferiores a 14\%, conforme trabalhos citados por WEIBEL \& VOS (1994) e nos estudos feitos por TREJO-CHANDIA et al. (1997).

Para VELLAME et al. (2010), o método de balanço de energia subestimou, em torno de 4,6\%, a transpiração de laranjeiras em relação ao método gravimétrico. Por outro lado, para pequenos intervalos de medição, WEIBEL \& VOS (1994), trabalhando com macieiras, observaram erros consideráveis entre o fluxo de seiva encontrado pelo método do balanço de energia e a transpiração medida. Considerando o intervalo de tempo de 30 min, o método de balanço de energia subestimou a transpiração em $20 \%$, enquanto o erro foi de apenas $4 \%$ para intervalos de 24 horas.

Quando dados de transpiração e do fluxo de seiva são analisados em escala diária, a transpiração da cana-de-açúcar foi subestimada em 56,2 $\mathrm{g}$ planta $^{-1} \mathrm{~d}^{-1}$, representando aproximadamente 7\% da transpiração máxima (Figura 6B). BAKER \& Van BAVEL (1987) sugerem que erros de até $10 \%$ são aceitáveis para estimativa de transpiração em plantas herbáceas, utilizando escalas de tempo de 2 a 24 horas.

TREJO-CHANDIA et al. (1997), analisando os erros de medição do fluxo de seiva de mudas de limoeiro, em comparação aos valores de transpiração obtidos em escala horária e no período diurno, compreendido entre 8 e $18 \mathrm{~h}$, constataram que o fluxo de seiva foi superestimado em 11,5\% e 13,3\%, respectivamente. Apesar da semelhança do erro percentual, foi observada maior dispersão dos dados em escala horária, em relação à integração dos valores para o período diurno.

De acordo com COELHO et al. (2012), a maior dispersão dos valores do fluxo de seiva, em relação aos de transpiração, é explicada pelo tempo de resposta do sistema planta-atmosfera diante do aumento ou da redução da demanda evaporativa. Assim, a transpiração obtida por meio de balanças de precisão não é identificada imediatamente pelo sistema de medição do fluxo de seiva no 
início da manhã. Em contraste, fato oposto é constatado no final do período diurno, quando a transpiração diminui abruptamente, e o fluxo de seiva continua para a reidratação do tecido vegetal. Portanto, verifica-se menor dispersão dos dados com a utilização de escalas de tempo maiores, pois a influência do tempo de resposta é amenizada ou eliminada.

A exatidão do método do balanço de energia também foi comprovada pelos altos valores do índice de concordância "d", que foram 0,$8987 ; 0,9355$ e 0,9432, para condições de céu nublado, parcialmente nublado e em ambas as condições de nebulosidade, respectivamente. Em escala diária, esse índice foi de 0,9488. Dessa forma, os resultados indicam que o método está adequadamente calibrado para medições em plantas de cana-de-açúcar.

Com relação à precisão das medições, avaliadas pelo RMSE, os valores obtidos foram 10,7 e 17,8 g planta $^{-1} \mathrm{~h}^{-1}$, para condições de céu nublado e parcialmente nublado, respectivamente (Figura 5). Os valores correspondentes do coeficiente de correlação " $\mathrm{r}$ " foram 0,8244 e 0,8958 , para condições de céu nublado e parcialmente nublado, respectivamente. Quando as duas condições de nebulosidade foram utilizadas na análise estatística, o valor do RMSE foi $14,6 \mathrm{~g} \mathrm{planta}^{-1} \mathrm{~h}^{-1}$, e o coeficiente de correlação, 0,9065. Já em escala diária, o RMSE foi $97,1 \mathrm{~g} \mathrm{planta}^{-1} \mathrm{~d}^{-1}$, e o coeficiente de correlação, 0,9369 .

\section{CONCLUSÕES}

As necessidades hídricas da cultura da cana-de-açúcar, em escala horária e diária, podem ser determinadas, de forma adequada, por meio de medições do fluxo de seiva com o método do balanço de energia, a partir da inserção de um minirresistor em um segmento do caule da planta.

Com base no indicador estatístico MBE, verificou-se que o método do balanço de energia apresentou desempenho satisfatório para a determinação da transpiração da cana-de-açúcar, tanto em condições de céu nublado como de parcialmente nublado, tendo subestimado a transpiração em torno de $5 \%$.

\section{AGRADECIMENTOS}

Esta pesquisa foi realizada com o apoio financeiro da Fundação de Amparo à Pesquisa do Estado de Minas Gerais (FAPEMIG) e do Conselho Nacional de Desenvolvimento Científico e Tecnológico $(\mathrm{CNPq})$. O primeiro autor também gostaria de agradecer à Universidade Federal de Viçosa, por meio do Programa de Pós-Graduação em Meteorologia Agrícola, pela oportunidade para aobtenção do título de Mestre.

\section{REFERÊNCIAS}

ALLEN, R.G.; PEREIRA, L.S.; HOWELL, T.A.; JENSEN, M.E. Evapotranspiration information reporting: I. Factors governing measurement accuracy. Agricultural Water Management, Amsterdam, v.98, p.899-920, 2011.

ALVES, E.L. Desenvolvimento de um sistema de medição dos fluxos de transpiração líquida para a cultura de cana-de-açúcar a partir do fluxo de seiva. 1999. 71 f. Dissertação (Mestrado em Engenharia Agrícola) - Universidade Estadual de Campinas, Campinas, 1999.

BAKER, J.M; Van BAVEL, C.H.M. Measurement of mass flow of water in the stems of herbaceous plants. Plant, Cell and Environment, Oxford, v.10, p.777-782, 1987.

CHABOT, R.; BOUARFA, S.; ZIMMER, D.; CHAUMONT, C.; MOREAU, S. Evaluation of the sap flow determined with a heat balance method to measure the transpiration of a sugarcane canopy. Agricultural Water Management, Amsterdam, v.75, p.10-24, 2005.

COELHO, R.D.; VELLAME, L.M.; FRAGA JÚNIOR, E.F. Estimation of transpiration of the 'Valencia' orange young plant using thermal dissipation probe method. Engenharia Agrícola, Jaboticabal, v.32, n.3, p.573-581, 2012. 
COSTA, R.F.; SILVA, V.P.R.; RUIVO, M.L.P.; MEIR, P.; COSTA, A.C.L.; MALHI, Y.S.; BRAGA, A.P.; GONÇALVES, P.H.L.; SILVA Jr., J.A.; GRACE, J. Transpiração em espécie de grande porte na Floresta Nacional de Caxiuanã, Pará. Revista Brasileira de Engenharia Agrícola e Ambiental, Campina Grande, v.11, n.2, p.180-189, 2007.

DELGADO-ROJAS, J.S.D.; ANGELOCCI, L.R.; FOLEGATTI, M.V.; COELHO FILHO, M.A. Desempenho da sonda de dissipação térmica na medida da transpiração de plantas jovens de lima ácida. Engenharia Agrícola, Jaboticabal, v.27, n.2, p.404-413, 2007.

DELGADO-ROJAS, J.S.D.; RIGUI, C.A.; KARASAWA, S.; ANGELOCCI, L.R.; BERNARDES, M.S.; FOLEGATTI, M.V. Desempenho do método de dissipação térmica na medida do fluxo de seiva em seringueira. Engenharia Agrícola, Jaboticabal, v.26, n.3, p.722-729, 2006.

GIRARDI, E.A.; MOURÃO FILHO, F.A.A.; DELGADO-ROJAS, J.S.; ARAÚJO, J.P.C. Use of the heat dissipation method for sap flow measurement in citrus nursery tree. Revista Brasileira de Fruticultura, Jaboticabal, v.32, n.4, p.976-983, 2010.

GOMIDE, R. L.; DURÃES, F.O.M.; KOBAYASHI, M.K.; MACHADO, R.A.F. Caracterização de estresse hídrico de duas linhagens de milho (Zea mays L.) com sondas de fluxo de seiva. Revista Brasileira de Milho e Sorgo, Sete Lagoas, v.4, n.3, p.344-354, 2005.

HERZOG, K.M.; THUM, R.; ZWEIFEL, R.; HÄSLER, R. Heat balance measurements - to quantify sap flow in thin stems only? Agricultural and Forest Meteorology, Amsterdam, v.83, p.7594, 1997.

IBGE - INSTITUTO BRASILEIRO DE GEOGRAFIA E ESTATÍSTICA. Sistema de Recuperação Automática - SIDRA. Disponível em:

$<$ http://www.sidra.ibge.gov.br/bda/agric/default.asp?t=2\&z=t\&o=11\&u1= $1 \& u 2=1 \& u 3=1 \& u 4=1 \& u 5=1 \& u 6=1>$. Acesso em: 25 nov. 2011.

IGBADUN, H.E.; MAHOO, H.F.; TARIMO, A.K.P.R.; SALIM, B.A. Crop water productivity of an irrigated maize crop in Mkoji sub-catchment of the Great Ruaha River Basin, Tanzania.

Agricultural Water Management, Amsterdam, v.85, p.141-150, 2006.

JACOVIDES, C.P.; KONTOYIANNIS, H. Statistical procedures for the evaluation of evapotranspiration computing models. Agricultural Water Management, Amsterdam, v.27, p.365371, 1995.

JALOTA, S.K.; SOOD, A.; CHAHAL, G.B.S.; CHOUDHURY, B.U. Crop water productivity of cotton (Gossypiumhirsutum L.) wheat (Triticumaestivum L.) system as influenced by deficit irrigation, soil texture and precipitation. Agricultural Water Management, Amsterdam, v.84, p.137146, 2006.

MACHADO, E.C.; OLIVEIRA, R.F.; RIBEIRO, R.V.; MEDINA, C.L.; STUCHI, E.S.; MARIN, F.R.; SILVA, J.A.B.; SILVA, S.R. Fluxo de seiva e fotossíntese em laranjeira 'Natal' com clorose variegada dos citros. Pesquisa Agropecuária Brasileira, Brasília, v.41, n.6, p.911-918, 2006.

MARIN, F.R.; RIBEIRO, R.V.; ANGELOCCI, L.R.; RIGHI, E.Z. Fluxo de seiva pelo método do balanço de calor: base teórica, qualidade das medidas e aspectos práticos. Bragantia, Campinas, v.67, n.1, p.1-14, 2008.

OLIVEIRA, G.X.S.; COELHO FILHO, M.A.; PEREIRA, F.A.C.; COELHO, E.F.; PAZ, V.P.S.; CASTRO NETO, M.T. Relações entre transpiração máxima, evapotranspiração de referência e área foliar, em quatro variedades de mangueira. Revista Brasileira de Fruticultura, Jaboticabal, v.31, n.1, p.20-27, 2009.

PIMENTEL, J.S.; SILVA, T.J.A.; BORGES Jr., J.C.F.; FOLEGATTI, M.V.; MONTENEGRO, A.A.A. Estimativa da transpiração em cafeeiros utilizando-se sensores de dissipação térmica. Revista Brasileira de Engenharia Agrícola e Ambiental, Campina Grande, v.14, n.2, p.187-195, 2010. 
RAJAK, D.; MANJUNATHA, M.V.; RAJKUMAR, G.R.; HEBBARA, M.; MINHAS, P.S. Comparative effects of drip and furrow irrigation on the yield and water productivity of cotton (Gossypiumhirsutum L.) in a saline and waterlogged vertisol. Agricultural Water Management, Amsterdam, v.83, p.30-36, 2006.

REIS, F.O.; CAMPOSTRINI, E.; SOUSA, E.F. Fluxo de seiva xilemática em mamoeiro 'Golden' cultivado por microaspersão sobre copa: relações com as variáveis ambientais. Bragantia, Campinas, v.68, n.2, p.285-294, 2009.

SAKURATANI, T. A heat balance method for measuring water flux in the stem of intact plantas. Journal of Agricultural Meteorology, Tokyo, v.37, n.1, p.9-17, 1981.

SMIT, M.A.; SINGELS, A. The response of sugarcane canopy development to water stress. Field Crops Research, Amsterdam, v.98, p.91-97, 2006.

TEJERA, N.A.; RODÉS, R.; ORTEGA, E.; CAMPOS, R.; LLUCH, C. Comparative analysis of physiological characteristics and yield components in sugarcane cultivars. Field Crops Research, Amsterdam, v.102, p.64-72, 2007.

TREJO-CHANDIA, J.E.; ANGELOCCI, L.R.; OLIVEIRA, R.F. Aplicação do método de balanço de calor na determinação da transpiração de mudas de limoeiro. Scientia Agricola, Piracicaba, v.54, n.3, p.1-16, 1997.

VASCONCELOS, L.F.L. Densidade de fluxo de seiva e relações hídricas foliares nas faces leste e oeste da copa de laranjeira 'Valência'. 2008. 115 f. Tese (Doutorado em Fisiologia e Bioquímica de Plantas) - Escola Superior de Agricultura "Luiz de Queiroz", Universidade de São Paulo, Piracicaba, 2008.

VELLAME, L.M.; COELHO FILHO, M.A.; PAZ, V.P.S. Transpiração em mangueira pelo método Granier. Revista Brasileira de Engenharia Agrícola e Ambiental, Campina Grande, v.13, n.5, p.516-523, 2009.

VELLAME, L.M.; COELHO FILHO, M.A.; PAZ, V.P.S.; COELHO, E.F. Stem heat balance method to estimate transpiration of young orange and mango plants. Revista Brasileira de Engenharia Agrícola e Ambiental, Campina Grande, v.14, n.6, p.594-599, 2010.

WEIBEL, F.P.; VOS, J.A.de. Transpiration measurements in apple trees: an improved stem balance heat method. Plant and Soil, The Hague, v.166, p.203-217, 1994.

ZHANG, Y.; KANG, S.; WARD, E.J.; DING, R.; ZHANG, X.; ZHENG, R. Evapotranspiration components determined by sap flow and microlysimetry techniques of a vineyard in northwest China: Dynamics and influential factors. Agricultural Water Management, Amsterdam, v.98, p.1.207-1.214, 2011.

ZOLNIER, S.; GATES, R.S.; GENEVE, R.L.; BUXTON, J.W. Evapotranspiration-based misting control for poinsettia propagation. Transactions of the ASAE, St. Joseph, v.46, n.1, p.135-145, 2003. 